\title{
Review: zotepine was as effective as typical and other atypical antipsychotics and more effective than placebo in schizophrenia
}

Fenton M, Morris S, De Silva Pet al. Zotepine for schizophrenia. Cochrane Review, latest version 27 Oct 1999. In: Cochrane Library. Oxford: Update Software.

QUESTION: In patients with schizophrenia and related psychoses, is zotepine effective compared with placebo and with typical and other atypical antipsychotic drugs?

\section{Data sources}

Studies were identified by searching 7 databases; by scanning bibliographies of identified articles; and by contacting authors of trials and the manufacturer of zotepine.

\section{Study selection}

Studies were selected if they were randomised controlled trials that compared zotepine with placebo or other antipsychotic agents in patients with schizophrenia or other psychoses.

\section{Data extraction}

Data were extracted on patient characteristics and follow up, setting, intervention, outcome measures (including global impression, mental state, leaving study early, and side effects), and methodological quality.

\section{Main results}

10 studies met the inclusion criteria. All outcomes were based on short term follow up (4-12 weeks).

2 trials compared zotepine with placebo. An 8 week trial $(n=106)$ favoured zotepine endpoint data for Clinical Global Impression (CGI) (mean difference [MD] in score $-1.0,95 \%$ CI -1.5 to -0.5), Scale for the Assessment of Negative Symptoms (SANS) (MD -12.5, CI -23 to -2), and for a reduction in Brief Psychiatric Rating Scale (BPRS) endpoint scores (table). Based on pooled data from 2 trials $(n=227)$ fewer patients taking zotepine dropped out, with an overall attrition rate of $60 \%$ (table).

7 trials $(n=670)$ compared zotepine with typical antipsychotics. 2 studies $(\mathrm{n}=135)$, when CGI endpoint scores were pooled, found improvements favouring zotepine (weighted MD -0.6, CI -1.0 to -0.2). Data from 4 trials $(n=356)$, using an a priori cut off point for improvement on the overall BPRS change score, favoured zotepine (table). Overall dropout rates did not differ significantly between patients treated with zotepine and typical antipsychotics (6 studies, $\mathrm{n}=437$ ).

2 trials $(n=230)$ investigated zotepine compared with a total of 4 other atypical antipsychotics. No clear differ- ences between these drugs were noted. Limited data were available.

\section{Conclusion}

In patients with schizophrenia and other related psychoses, zotepine was more effective than placebo, at least as effective as typical antipsychotic drugs, and not clearly different from atypical antipsychotic drugs.

\section{COMMENTARY}

The current literature on second generation or "atypical" antipsychotic drugs has investigated at least 3 potential advantages: (1) improved efficacy on more traditional measures such as positive and negative symptoms; (2) a broader spectrum of efficacy (eg, cognitive, affective symptoms); and (3) an improved side effect/tolerability profile.

The present data on zotepine, albeit limited, in this review by Fenton et al suggest zotepine too may share some of these features and might be used with these goals in mind. Having said this, however, various issues relevant to the atypical agents in general, and therefore zotepine, warrant consideration. Firstly, atypical agents are best viewed along a continuum rather than as a dichotomous variable that groups them as identical and in contrast to typical antipsychotics. Secondly, various studies comparing atypical with typical antipsychotics have used inappropriately high doses of the typical agents for the purpose of comparison, which in turn will influence outcome measures such as negative symptoms, extrapyramidal symptoms (EPS), and cognition. This is true for at least some of the zotepine data. Thirdly, results will be influenced by symptom phase (acute, chronic) and stage (early eg, first episode; chronic; refractory). Controlled data for zotepine are lacking for specific subpopulations (eg, first episode, truly refractory). Fourthly, distinguishing the newer antipsychotics along a broader spectrum of outcome measures (eg, cognition, quality of life) is in its early stages. Controlled data specific to zotepine on these dimensions are lacking or too preliminary to draw firm conclusions. Fifthly, the problem of weight gain and associated consequences with the newer antipsychotic agents may turn this adverse event into the "EPS" of these second generation antipsychotic agents. More detailed information on zotepine in this respect is required.

For some patients with schizophrenia and related psychosis, available evidence may support using atypical antipsychotics to replace their conventional counterparts. How to choose among this growing number of compounds is less than clear, however. Initial reports suggest clinical comparability between zotepine and at least some of the other atypical agents, but once again the limited controlled data available make it impossible to draw firm conclusions or recommend guidelines at this time.

Gary Remington, $\mathrm{MD}, \mathrm{PhD}, \mathrm{FRCP}(\mathrm{C})$ Centre for Addiction And Mental Health, Clark Division Toronto, Ontario, Canada 